\title{
Modulating human sense of agency with non-invasive brain stimulation
}

2

3 Nima Khalighinejad, Patrick Haggard

4 Institute of Cognitive Neuroscience, University College London, London WC1N 3AR, UK

5

6 Correspondence should be addressed to Nima Khalighinejad, Institute of Cognitive Neuroscience,

7 University College London, Alexandra House, 17 Queen Square, London WC1N 3AR, UK. Tel: +44

8 (0)20 7679 5434, E-mail: nima.khalighinejad.12@ucl.ac.uk

9

10 Running title: Human sense of agency 


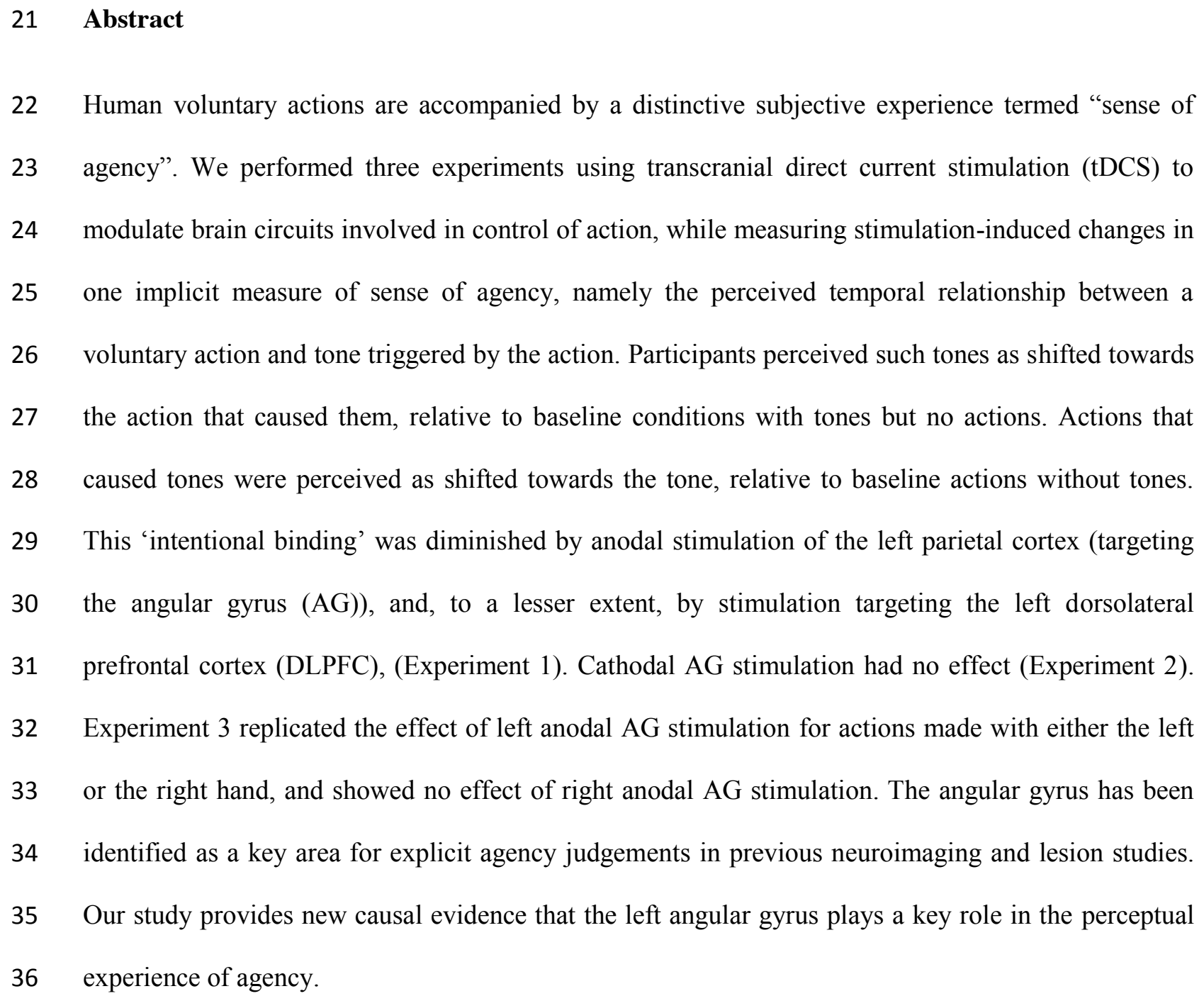

22 Human voluntary actions are accompanied by a distinctive subjective experience termed "sense of 23 agency". We performed three experiments using transcranial direct current stimulation (tDCS) to 24 modulate brain circuits involved in control of action, while measuring stimulation-induced changes in 25 one implicit measure of sense of agency, namely the perceived temporal relationship between a 26 voluntary action and tone triggered by the action. Participants perceived such tones as shifted towards 27 the action that caused them, relative to baseline conditions with tones but no actions. Actions that caused tones were perceived as shifted towards the tone, relative to baseline actions without tones.

29 This 'intentional binding' was diminished by anodal stimulation of the left parietal cortex (targeting 30 the angular gyrus (AG)), and, to a lesser extent, by stimulation targeting the left dorsolateral 31 prefrontal cortex (DLPFC), (Experiment 1). Cathodal AG stimulation had no effect (Experiment 2). 32 Experiment 3 replicated the effect of left anodal AG stimulation for actions made with either the left 33 or the right hand, and showed no effect of right anodal AG stimulation. The angular gyrus has been 34 identified as a key area for explicit agency judgements in previous neuroimaging and lesion studies. 35 Our study provides new causal evidence that the left angular gyrus plays a key role in the perceptual 36 experience of agency.

37 Keywords: sense of agency; angular gyrus; dorsolateral prefrontal cortex; intentional binding; tDCS 


\section{Introduction}

46 Healthy human adults have the feeling that they are able to control their own actions, and, through

47 them, external events. This is referred to as the sense of agency. Sense of agency is central to individual goal-directed action, and also to social responsibility and punishment (Frith, 2014; Moretto,

49 Walsh, \& Haggard, 2011). Moreover, many neurological and psychiatric disorders involve

50 abnormalities of agency (de Jong, 2011; Fletcher \& Frith, 2009; Kranick \& Hallett, 2013).

51 Despite extensive theoretical work on agency, its neural correlates are not fully understood.

52 Neuroimaging studies found activation of AG (Farrer et al., 2003; Farrer \& Frith, 2002; Farrer et al., 53 2008) and DLPFC (Fink et al., 1999) associated with agency tasks, but the activation of these areas 54 was always greater in the conflicting, non-agency condition than in the agency condition. In a recent meta-analysis of sense of agency, the single most consistent result was activation of a broadly-defined temporoparietal junction area conditions associated with reduced or absent sense of agency (Sperduti, 57 Delaveau, Fossati, \& Nadel, 2011). This broad 'non-agency' area includes AG. Computational models of predictive motor control offer an important theoretical framework for understanding agency. An internal forward model uses efference copies of the motor command to predict outcomes 60 (Wolpert \& Ghahramani, 2000). According to these models, sense of agency arises when there is a match between the predicted and actual sensory outcome of the generated action. Conversely, if current sensory information does not match the model's prediction, then the corresponding sensory event cannot be self-generated, and no sense of agency is experienced (Frith, Blakemore, \& Wolpert, 64 2000).

65 Farrer et al., (2008) used this framework to interpret fMRI activations of AG in particular, suggesting that $\mathrm{AG}$ processes discrepancies between intended action and its actual consequences. Her data

67 showed increased activations of AG when a detectable temporal discrepancy was inserted between an action and visual feedback of the outcome, and also when participants explicitly rejected agency over 69 the viewed outcome. 
Most of these studies used explicit agency attribution tasks, in which participants judge whether they

71 did or did not cause a specific sensory event. Synofzik, Vosgerau, \& Newen, (2008) noted that one

72 feels a sense of agency when acting, even without making any explicit judgements. One suitable measure of this pre-reflective, sensorimotor feeling of agency is the perceived temporal relationship between a voluntary action and its sensory outcome (Moore \& Obhi, 2012). The perceived time of voluntary actions and their sensory consequences are attracted towards each other. This 'intentional binding' is absent, or less prominent, for involuntary movements, and for associations between external events not involving voluntary actions (Cravo, Claessens, \& Baldo, 2009).

The neural bases of such feelings of agency are poorly understood. One neuroimaging study found a neural correlate of intentional binding in the medial frontal cortex (Kühn, Brass, \& Haggard, 2013). A

'virtual lesion' study showed that theta-burst stimulation over a slightly more anterior medial frontal location reduced the intentional binding effect (Moore, Ruge, Wenke, Rothwell, \& Haggard, 2010). On the other hand, other lesion (Sirigu et al., 2004) and stimulation (Desmurget et al., 2009) studies suggested an important role of parietal cortex in intentional action and agency, though these studies did not use binding. To our knowledge, no previous causal study has investigated the influence of both frontal and parietal areas on sense of agency using implicit measures. We therefore performed three transcranial direct current stimulation (tDCS) experiments, to modulate excitability of key brain circuits underlying the control of action, while measuring the effects on sense of agency, using intentional binding. Our experiments investigated the respective contributions of parietal and frontal areas to intentional binding as a proxy measure of agency (Experiment 1), their susceptibility to both up- and down-regulation (Experiment 2), and their hemispheric specialisation (Experiment 3).

Based on the existing neuroimaging data investigating explicit agency judgement (Farrer et al., 2003; Farrer \& Frith, 2002; Farrer et al., 2008), we predicted that anodal stimulation of putative AG should also influence the sense of agency, as measured by intentional binding. Importantly, such a result would identify a causal role for AG in sense of agency, but would not conclusively identify how AG computes agency. We also investigated the role of prefrontal areas in sense of agency. Studies of frontal contributions to sense of agency are more equivocal. Neurostimulation (Moore et al., 2010) 
and neuroimaging (Kühn et al., 2013) studies of intentional binding found evidence for medial

98 prefrontal involvement, but studies of explicit agency judgements in tasks requiring a choice between 99 alternative actions (Chambon, Wenke, Fleming, Prinz, \& Haggard, 2013) identified a more lateral 100 prefrontal focus. DLPFC has also been identified as a key area for initiation (Jahanshahi et al., 1995) 101 and monitoring of voluntary action (Rowe, Hughes, \& Nimmo-Smith, 2010). Given the relative inaccessibility of medial prefrontal cortex to neurostimulation, we focussed here on the lateral prefrontal cortex. The stimulations targeted primarily the left hemisphere, and participants made actions with their right hand (experiments 1,2), or with either hand (experiment 3).

\section{Materials and Methods}

\subsection{Participants}

In total 55 healthy volunteers, $18-35$ years of age ( 25 females) were recruited from the Institute of Cognitive Neuroscience subject data pool for three separate experiments. All participants were right handed, had normal or corrected to normal vision, had no history or family history of seizure, epilepsy or any neurologic or psychiatric disorder and did not have any metallic or electronic object in the head. Participants affirmed that they had not participated in any other brain stimulation experiment in the last 48 hours, nor had consumed alcohol in the last 24 hours. The sample for Experiment 1 consisted of 18 participants ( 8 females), Experiment 2 consisted of 19 participants (10 females) and Experiment 3 consisted of 18 participants ( 7 females). One participant failed to finish Experiment 2 due to lack of concentration, and was therefore excluded. Experimental design and procedure were approved by the UCL research ethics committee, and followed the principles of the Declaration of Helsinki. Transcranial stimulation followed established safety procedures (Nitsche et al., 2003; Poreisz, Boros, Antal, \& Paulus, 2007). Participants were paid a minimum amount for participating in each session of the experiment. Participants were paid a small additional bonus at the end of the last session.

2.2. Behavioural task 
We used intentional binding paradigm as an implicit measure of agency (Fig. 1). The task was based on previous studies (Haggard, Clark, \& Kalogeras, 2002), and was programmed in LabVIEW 2012 (Austin, Texas). Participants viewed a clock hand rotating on a computer screen which was located $60 \mathrm{~cm}$ in front of the participants in a quiet room. The initial clock position was random. Clock rotation was initiated by participants pressing the return key on a keyboard. Each full rotation lasted 2560ms. Participants were instructed to look at the centre of the clock. They made voluntary actions, when instructed, by pressing the return key with their right index finger (Experiments 1,2), or by pressing F9 or F4 with their right or left index finger, respectively (Experiment 3). Participants chose for themselves when to make these voluntary actions. After each key press, the clock hand stopped at a random location, participants made a time judgement according to condition (see later). Each experimental session consisted of four types of trials, presented in separate blocked and randomised conditions. At the beginning of each block, brief instructions for the relevant condition were displayed on the screen. In the baseline action condition, participants had to press the key at a time of their own free choice. The clock hand stopped after 1500-2500ms (at random), and participants then judged the clock hand position at the time of their key press, entering their response on the keyboard. In this condition, the participant's actions produced no sensory outcome. In the baseline tone condition, participants were instructed to look at the clock but not to press any key. While the clock was rotating, a pure tone $(1000 \mathrm{~Hz}, 100 \mathrm{~ms}$ duration) was played over a loudspeaker, $1750-4000 \mathrm{~ms}$ (at random) after the onset of the trial. Participants were then asked to judge the clock hand position at the time of the tone. In the operant action condition, participants pressed a key at a time of their own choosing, and each keypress produced a tone after $250 \mathrm{~ms}$. Participants judged the clock hand position at the moment of pressing the key. Finally, the operant tone condition was similar to the operant action condition, with the difference that participants had to judge the clock hand position at the time of the tone. Each condition was tested in a separate block of 30 trials. The order of the blocks was randomised and there was a 1 minute break between each block.

This common basic design was slightly changed according to the demands of each specific experiment. In Experiment 3, text below and above the clock instructed participants to reply with 
either their left or right index fingers. The order of hands was randomised in each block. The block length was increased by 40 trials to allow sufficient trials for analysis of each hand's data.

Before each experiment, participants were trained and familiarised with the task. They were reminded to look at the centre of the clock, to avoid following the clock hand with their eyes, to be spontaneous in their key presses and to be as precise as possible in their judgements, in particular not confining themselves to those numbers $5,10,15 \ldots$ marked on the clock face. Each experimental session consisted of four blocks and took approximately 20 minutes. The short duration of each individual session was planned to coincide with the known effective period of tDCS.

\section{3. tDCS}

Direct current stimulation was delivered by StarStim noninvasive wireless neurostimulator (Neuroelectrics, Barcelona, Spain). Circular rubber electrodes $\left(25 \mathrm{~cm}^{2}\right)$ were covered in saline-soaked sponges, installed in a 27 channel neoprene cap, and connected to a wireless current generator. tDCS was then controlled by Neuroelectrics Instrument Controller (NIC v1.2) through a separate computer. Current strength was set at $1 \mathrm{~mA}$ in all experiments, generating a current density of $0.04 \mathrm{~mA} / \mathrm{cm}^{2}$ at the scalp surface. For each experiment, all participants underwent three separate sessions of tDCS, two effective stimulations and one sham session. The order of the sessions was randomised and counterbalanced across participants. There was a minimum of 48 hours (and a maximum of 1 week) between each stimulation session to minimise any potential carry over effects of tDCS (Nitsche et al., 2008). The duration of stimulation in each session was set at 25 minutes, including 30s to ramp-up and down the stimulating current. For the sham condition, electrical current was only applied during the first and last 30 seconds of the stimulation, so as to induce the same cutaneous sensation as real stimulation, and thus blind the participants as to stimulation condition. During the first $5 \mathrm{~min}$ of each stimulation, participants were asked to relax on their seats and close their eyes. This delay was designed to allow potential neuro-modulatory effects to build up (Zwissler et al., 2014). Next, participants began the behavioural task while stimulation continued (Fig. 2). All participants finished the behavioural task approximately after $20 \mathrm{~min}$, the same time as the end of stimulation. In case participants finished the task prior to the end of stimulation they were asked to remain seated until the 
end of the stimulation. In case the task outlasted the stimulation, they continued to perform the task without further stimulation. The task period never exceeded the stimulation period by more than 2 minutes.

Fig. 2 shows tDCS montages of the three experiments. In Experiment 1, the anodal electrode was placed on the left DLPFC (F3 according to the 10/20 international EEG electrode placement) or putative left AG (position P3) (Okamoto et al., 2004; Spitoni et al., 2013) in separate sessions. During the sham session, the position of the stimulating electrode was counterbalanced between F3 and P3. In all three sessions, the return electrode (cathodal) was placed on the right supraorbital area. For Experiment 2, anode and cathode were placed on the putative left AG in separate sessions while the return electrode was placed on the right supraorbital area. This arrangement was retained during the sham session. Experiment 3 used a biparietal montage (Cohen Kadosh, Soskic, Iuculano, Kanai, \& Walsh, 2010; Hecht, Walsh, \& Lavidor, 2010). For anodal stimulation of the putative left AG, the anode was placed over P3 and cathode was placed over P4. This arrangement was reversed for anodal stimulation of the putative right AG. For sham stimulation, the anode was pseudorandomly placed either at P3 or P4. After each session participants were asked as part of debriefing if they had experienced any notable effects of stimulation. No effects were reported other than mild tingling sensations localised to the electrodes.

\subsection{Data analysis}

The difference between the judged clock hand position and the actual onset of the corresponding event was calculated, giving a judgement error for each trial. A perceptual delay was represented by a positive judgement error, and an anticipation by a negative judgement error. The mean and standard deviation of the judgement errors across trials were then measured for each condition. Action binding was defined as the shift of action toward its outcome, and was calculated by subtracting each participant's mean judgement error in the baseline action from that in the operant action condition. Likewise, tone binding was defined as a shift in the perceived time of a tone towards the action that caused it. Tone binding was calculated by subtracting each participant's mean judgement error in 
202

baseline tone condition from that in the operant tone condition. Thus, perceptual association of an

203 action with a subsequent tone produced a positive value for action binding, and a negative value for 204 tone binding. We analysed action and tone binding separately, since there is evidence from both 205 cognitive studies (Wolpe, Haggard, Siebner, \& Rowe, 2013), and previous neurostimulation studies 206 (Moore et al., 2010), that they are driven by distinct mechanisms.

207 Some participants were excluded because of highly variable time judgement. A standard deviation of 208 judgement error across trials of over $250 \mathrm{~ms}$ in any condition was used as a marker of poor time 209 perception. As in previous intentional binding experiments (Haggard, Aschersleben, Gehrke, \& Prinz, 210 2002), these participants were excluded. On this basis, two participants were excluded from 211 Experiment 1, two from Experiment 2, and four from Experiment 3. Importantly, these exclusion 212 criteria are orthogonal to the mean judgement errors used for statistical inference.

213 In Experiments 1 and 2, inferential statistics were based on one-way repeated measures ANOVA, 214 with paired-sample t-tests for follow-up testing. Because our ANOVA had only 3 levels, Bonferroni correction was not required for follow-up testing after a significant result (Cardinal \& Aitken, 2013; Meier, 2006). Additionally, in Experiment 1, we used linear discriminant analysis to determine which percepts were most strongly affected by stimulation condition. Experiment 3 used repeated measures ANOVA, with the additional factor of acting hand (right hand responses vs. left hand responses). A final pooled analysis was performed to compare effects of stimulation common to all conditions.

\section{Results}

3.1. Experiment 1: frontal vs parietal anodal stimulation

This experiment compared the effects of frontal (targeting left DLPFC) and parietal (targeting left AG) cortex stimulation on intentional binding for actions and tones. One-way repeated measuresANOVA with the factor of stimulation type (anodal frontal vs. anodal parietal vs. sham) showed that action binding was not significantly affected by the type of stimulation $(F(2,30)=1.90, p=0.17$, $\left.\eta^{2}=0.11\right)$. However, an identical ANOVA on tone binding showed significant differences $(\mathrm{F}(2$, $30)=4.30, p=0.02, \eta^{2}=0.22$ ). Follow-up testing showed that tone binding was significantly reduced by 
anodal stimulation of the putative left AG compared to sham $(\mathrm{t}(15)=2.67, \mathrm{p}=0.02, \mathrm{~d}=0.43)$. Anodal stimulation of the left DLPFC showed a clear trend to reduce tone binding, which approached the border of conventional significance $(\mathrm{t}(15)=2.07, \mathrm{p}=0.06, \mathrm{~d}=0.42)$. There was no significant difference between the frontal and parietal stimulation $(\mathrm{t}(15)=-0.10, \mathrm{p}=0.92, \mathrm{~d}=0.02)$ (Fig. 3; see also supplementary table A.1-6).

233 We additionally performed the same analysis using median rather than mean judgement error in each condition (see supplementary table B.1-3), since median measures are more robust than means to the influence of outliers. The patterns of statistical significance were unchanged.

Finally, to confirm that anodal stimulation of putative AG affected primarily the operant tone condition and not the baseline tone, the effect of stimulation type on participants' judgement error in baseline tone conditions was assessed using repeated-measure one-way ANOVA. There was no significant main effect of stimulation type on baseline tone condition $(F(2,30)=0.58, p=0.56$, $\left.\eta^{2}=0.04\right)$. This suggests that stimulation influenced a neurocognitive process that is present primarily in the operant condition.

We additionally applied multivariate linear discriminant analysis (Krzanowski, 2000) to identify the linear combination of action binding and tone binding variables that optimally discriminates the different stimulation conditions. Linear discriminant analysis significantly differentiated the three stimulation conditions (Wilks' Lambda $=0.59$, approx. $\mathrm{F}(4,58)=4.36, \mathrm{p}<0.01$ ). Inspection of canonical coefficients showed that this difference was primarily due to tone binding (standardized canonical coefficient 1.86) rather than action binding $(-0.93)$ (The scores of the individual participants on the first discriminant variate are shown in supplementary Fig. A.1). Post-hoc comparisons between conditions showed a highly significant difference between parietal and sham stimulation $(\mathrm{p}<0.01$; standardised coefficients -1.03 for action binding, 2.19 for tone binding), and also a significant difference between frontal and sham stimulation ( $\mathrm{p}=0.04$; standardised coefficients -0.82 for action binding, 1.55 for tone binding). Interestingly, the frontal effect thus involved a slightly larger action binding coefficient, considered relative to the tone binding coefficient, than did the parietal effect, 
254

though no inferential statistics can be applied to this ratio. Frontal and parietal stimulation did not differ significantly $(\mathrm{p}=0.74)$.

\subsection{Experiment 2: anodal vs cathodal parietal stimulation}

257 If anodal stimulation boosts activity in the left AG then cathodal stimulation of the same area should 258 lead to its suppression. Thus, if anodal stimulation decreases intentional binding, cathodal stimulation 259 should increase it. To test this hypothesis, putative left AG was exposed to anodal, cathodal or sham 260 stimulation in different sessions, and effects on intentional binding were evaluated.

261 One-way repeated measures-ANOVA with the factor of stimulation type (anodal parietal vs. cathodal parietal vs. sham) was used for analysis. Action binding was not significantly affected by the type of stimulation $\left(\mathrm{F}(2,30)=0.50, \mathrm{p}=0.61, \eta^{2}=0.03\right)$. Tone binding was also unaffected by type of stimulation $\left(F(2,30)=0.45, p=0.64, \eta^{2}=0.03\right)$, contrary to our predictions from Experiment 1 . Nevertheless, the numerical effect of anodal stimulation of the putative left AG was in the same direction as Experiment 1, namely a decreased tone binding compared to sham and cathodal stimulation (Fig. 4; see also supplementary table A.7-12).

We additionally performed the same analysis using median rather than mean judgement error in each condition (see supplementary table B.4-6). The patterns of statistical significance were unchanged.

\subsection{Experiment 3: left vs right parietal stimulation}

Experiment 3 aimed to replicate the effects of Experiment 1, and additionally investigated the lateralisation of intentional binding using a biparietal montage. The biparietal montage may provide a higher local current density, because of the relatively short path between anode and cathode. In addition, this montage controls for any possible effect of cathodal stimulation of prefrontal areas that may occur with the conventional supraorbital placement of the cathode. Therefore, putative left and right AG were exposed to anodal stimulation in separate sessions, and a third session involved sham stimulation. We also investigated whether the putative AG involvement in intentional binding is handspecific or hemisphere-specific, by asking participants to make actions with either the left or right 
hand, choosing randomly on each trial. Analysis of action binding showed no significant main effect of stimulation type $\left(F(2,26)=0.06, p=0.94, \eta^{2}=0.01\right)$ or acting hand $\left.F(1,13)=0.10, p=0.76, \eta^{2}=0.01\right)$ and no significant interaction $\left(F(2,26)=0.89, p=0.42, \eta^{2}=0.06\right)$. Analysis of tone binding showed a highly significant main effect of stimulation $\left(F(2,26)=5.93, p<0.01, \eta^{2}=0.31\right)$. Follow-up testing showed that anodal stimulation of the putative left AG significantly decreased tone binding relative to both sham stimulation $(\mathrm{t}(13)=2.55, \mathrm{p}=0.02, \mathrm{~d}=0.40)$, and relative to anodal stimulation of the putative right $\mathrm{AG}(\mathrm{t}(13)=2.90, \mathrm{p}=0.01, \mathrm{~d}=0.56)$. No significant difference was observed between anodal stimulation of the putative right $\mathrm{AG}$ and sham $(\mathrm{t}(13)=-0.75, \mathrm{p}=0.47, \mathrm{~d}=0.10)$ (Fig. 5; see also supplementary table A.13-18). Acting hand had no significant main effect on tone binding $(\mathrm{F}(1$, 13) $\left.=0.01, p=0.94, \eta^{2}<0.01\right)$ and no interaction was observed between the stimulation and acting hand $\left(\mathrm{F}(2,26)=0.15, \mathrm{p}=0.86, \eta^{2}=0.01\right)$.

We additionally performed the same analysis using median rather than mean judgement error in each condition (see supplementary table B.7-9). The patterns of statistical significance were unchanged.

To check whether the decrease in tone binding was primarily due to shifts in the operant tone, or in the baseline tone condition, participants' judgement errors in the baseline tone condition were compared across the stimulation groups. Analysis showed no significant main effect of stimulation type on baseline tone condition $\left(\mathrm{F}(2,26)=0.50, \mathrm{p}=0.60, \eta^{2}=0.04\right)$.

\subsection{Pooled data: anodal parietal vs sham stimulation}

Anodal stimulation of the putative left AG was common to all three experiments reported here, as was a sham stimulation condition, although the experiments differed in other respects. Therefore, we pooled the data in these specific conditions across the 46 participants ( 21 female) from the three experiments, in a single analysis. We found that anodal stimulation of putative left AG significantly reduced the perceptual shift of tone toward action compared to sham $(\mathrm{t}(45)=3.28, \mathrm{p}<0.01, \mathrm{~d}=0.35)$, but had no effect on action binding $(\mathrm{t}(45)=-1.37, \mathrm{p}=0.18, \mathrm{~d}=0.22)$.

The anodal montage in the third session used a different return (cathode) location compared to the 304 first and second experiments. Our decision to pool data of the three studies was based on the common 
placement of the anode across these three experiments, and the general observation that cathodal

306 effects on cognitive function are rare (Jacobson, Koslowsky, \& Lavidor, 2012). In that case,

307 differences in cathode location may be relatively unimportant, and need not prevent pooling across

308 studies. However, because we cannot entirely exclude some contribution of cathodal location to our

309 main results, we ran a further pooled analysis using the left anodal stimulation conditions of

310 experiments 1 and 2 only, which share a supraorbital cathode location, but excluding experiment 3 .

311 This analysis again found that anodal stimulation of putative left AG significantly reduced the 312 perceptual shift of tone toward action compared to sham $(\mathrm{t}(31)=2.45, \mathrm{p}=0.02, \mathrm{~d}=0.35)$, but had no 313 effect on action binding $(\mathrm{t}(31)=-1.48, \mathrm{p}=0.15, \mathrm{~d}=0.32)$.

314 To investigate the generality of the anodal AG effect across experiments, we performed a mixed 315 ANOVA with a between-subject factor of experiments (1,2 or 3), and a repeated-measures factor of 316 stimulation type (anodal left AG vs. sham). The main effect of stimulation type $(F(1,43)=10.7$, $\left.317 \mathrm{p}<0.01, \eta^{2}=0.20\right)$ recapitulating the pooled t-test reported above. There was no significant main effect 318 of experiment $\left(F(2,43)=0.80, p=0.45, \eta^{2}=0.04\right)$. Importantly, there was no hint of interaction between 319 experiment and stimulation $\left(\mathrm{F}(2,43)=0.96, \mathrm{p}=0.39, \eta^{2}=0.04\right)$.

320 Our main inferences above are based on comparing experimental stimulation with sham. We therefore 321 additionally investigated whether sham stimulation had different effects in the three experiments. We 322 found no significant difference among the sham conditions for the 3 experiments for action binding $323(\mathrm{~F}(2,43)=2.20, \mathrm{p}=0.12)$, or tone binding $(\mathrm{F}(2,43)=1.10, \mathrm{p}=0.33)$.

\section{4. Discussion}

325 4.1. Stimulation-induced modulation of the left parietal cortex

326 We performed a series of three tDCS experiments to investigate the neural circuits responsible for the sense of agency, as measured by the perceptual association between the time of a voluntary action and the time of a resulting auditory tone. We found a significant decrease in the binding of outcomes towards actions after anodal stimulation of the putative left AG (Experiment 1). Anodal stimulation of 330 the left DLPFC also decreased action and tone binding compared to sham. DLPFC affected tone 
binding as much as AG stimulation, but its effects were less consistent across participants.

332 Nevertheless, our discriminant analysis showed a significant effect of DLPFC stimulation compared 333 to sham, when action and tone binding were considered together.

334 Our tDCS stimulation of putative AG could have widespread effects across the inferior parietal cortex 335 (IPC), since tDCS has quite low spatial specificity. For example, anterior parts of the IPC may also be 336 affected. IPC is routinely activated in neuroimaging studies when participants judge whether their 337 own action, or some other cause, is responsible for a specific sensory event. In a study by Farrer and 338 Frith (2002), the IPC area was more active when participants attributed a visual event to another 339 person, rather than to themselves. Similarly, a PET study (Farrer et al., 2003) observed that neural 340 activity in IPC increased with the level of discrepancy between the executed and the observed action 341 on the screen. In an fMRI study (Farrer et al., 2008), the subjective feeling of loss of control 342 correlated with BOLD response in the AG, as did the awareness of temporal discrepancy between 343 action and feedback. The authors of those studies suggested that AG houses the comparison between 344 the efference copy of the intended action and the actual sensory outcome. Any mismatch between 345 these signals will then give rise to the explicit awareness of non-agency, or an external source of 346 action.

347 Our overall results are consistent with this view. We found that anodal stimulation of the putative AG 348 decreases intentional binding, our proxy measure for agency. Anodal stimulation is generally thought 349 to increase the activity of the cortical region immediately under the electrode. However, AG 350 activation is routinely associated with lack of agency, rather than with experience of positive agency 351 (Farrer \& Frith, 2002; Farrer et al., 2008; Sperduti et al., 2011). Therefore, excitation of a neural 352 substrate of non-agency might be expected to decrease intentional binding. The conventional polarity353 specific (anode-boosting, cathode-suppressing) framework of tDCS was developed on the basis of 354 effects in primary motor cortex stimulation (Nitsche \& Paulus, 2000). Its applicability to non-primary 355 areas and cognitive processing has recently been questioned (Horvath, Forte, \& Carter, 2014). 356 Nevertheless, our findings are broadly compatible with the conventional polarity-specific view. 
Since DLPFC is normally thought to facilitate intentional action, one may question why prefrontal anodal tDCS did not increase intentional binding. Rowe et al., (2010) questioned whether DLPFC played any important role in initiation of simple voluntary actions, such as those tested here, and suggested a role in monitoring sequential action patterns instead. Fink et al., (1999) observed 362 activation of DLPFC using PET when an intentional action and its sensory outcome were 363 incompatible. Anodal tDCS over DLPFC might correspond to an increased coding for action-outcome 364 conflict, even though our task did not explicitly manipulate action-outcome compatibility. Our 365 discriminant analysis found some evidence consistent with this interpretation. However, this effect but not in univariate analyses of action binding and tone binding separately. Therefore, further research is required before a strong statement about frontal tDCS effects on sense of agency can be made.

\subsection{Polarity-specific effects of tDCS}

Experiment 2 aimed to investigate whether parietal stimulation effects were polarity-specific. On one model, tDCS would simply add neural noise, irrespective of polarity. On another model, anodal stimulation would upregulate putative non-agency coding in AG, while cathodal stimulation should down-regulate it. The result of anodal left AG stimulation in experiment 2 followed the expected trend for tone binding, but did not reach statistical significance. Replication of statistically significant results is an important and controversial issue in modern neuroscience (Cumming, 2005). All effects measured in experiments represent a combination of the underlying 'true' effect, and noise. Importantly, when a nonzero true effect indeed exists, but is modest in size, it is quite likely for the effect to reach statistically significant levels in one study, but not in another. Thus, absence of a significant anodal tDCS effect in experiment 2 does not prove that no true effect exists: we return to this point later. 
382

Experiment 2 found no significant difference between cathodal and sham stimulation, although we had predicted that cathodal stimulation might enhance intentional binding. Although inhibitory cathodal effects on motor function are well established, a recent review of 34 studies found that cathodal inhibitory effects on cognitive function are rare (Jacobson, Koslowsky, et al., 2012). Another possible reason for the absence of any significant cathodal AG effect in Experiment 2 could be the placement of the anode electrode on the supraorbital area. This location is standard for tDCS studies of action (Nitsche et al., 2008). However, it causes a strong current density close to the frontopolar and prefrontal areas, where the anode is located. These areas may also contribute to intentional action (Brass \& Haggard, 2007). Thus, our montage for cathodal stimulation of AG in experiment 2 involved anodal stimulation at a frontopolar site, which may not be strictly neutral for sense of agency. Future studies could address this issue by using extracephalic cathode placement.

\subsection{Hemispheric specialisation of the sense of agency}

394 Experiment 3 avoided the potential confound of frontopolar stimulation using a biparietal montage. 395 This produces a higher current density in a small region surrounding the electrodes (Cohen Kadosh et 396 al., 2010; Nathan, Sinha, Gordon, Lesser, \& Thakor, 1993), compared to the conventional supra397 orbital location. This might result in a more focal stimulation. More importantly, the biparietal 398 montage excludes the possibility that the significant effects of anodal AG stimulation in experiments 3991 and 2 were in fact caused by cathodal frontopolar stimulation. Specifically, if the effects in 400 experiments 1 and 2 were merely due to cathodal frontopolar stimulation, then no effect of stimulation 401 should be found in experiment 3. The biparietal montage also allowed us to investigate lateralisation 402 of agency by varying both tDCS polarity and the hand used for action. Similar approaches have been 403 used previously in other studies (Bardi, Kanai, Mapelli, \& Walsh, 2013; Jacobson, Goren, Lavidor, \& 404 Levy, 2012).

405 The results of the third experiment replicated our previous findings. Anodal stimulation of putative 406 left AG significantly decreased tone binding compared to both sham and cathodal stimulation of the 
same area. The tDCS effect was statistically equivalent whether the action was made with the left or

the right hand. No effects were observed with anodal stimulation of the putative right AG.

Experiment 3 does not support the alternative interpretation of experiments 1 and 2 based on a putative cathodal frontopolar stimulation. In contrast, experiment 3 supports the interpretation of an anodal left AG effect. We cannot conclusively rule out some contribution of frontopolar stimulation to our results, but we can rule in a specific contribution of the left AG.

413 Experiment 3 adds several important elements to the previous studies. First, it demonstrates an 414 involvement of AG in a task involving randomised, stimulus-driven selection between alternative 415 actions, as opposed to mere repetition of a simple action. Second, it suggests that left, but not right 416 AG is responsible for action-outcome binding for actions made by either hand. We found no 417 interaction between stimulation and hand used for action. Previous neuroimaging studies have 418 reported activation corresponding to non-agency judgements in both left and right AG. Interestingly, 419 right AG activations appeared to dominate (Farrer et al., 2003; Farrer \& Frith, 2002; Farrer et al., 420 2008), in contrast to our finding. However, in a more recent fMRI study, Lee \& Reeve, (2013) 421 reported higher activity in the left AG during non-self-determined behaviour, consistent with our 422 hypothesis in Experiment 1 that anodal AG stimulation activates a neural code for 'non-agency'. 423 Finally, hemispheric specialisation of agency could plausibly depend on the task used, and the type of 424 agency judgement. Previous neuroimaging studies generally used explicit judgements of agency, and 425 often used complex manual actions with visual feedback (Farrer \& Frith, 2002; Farrer et al., 2008; 426 Sperduti et al., 2011). We are not aware of any neuroimaging study investigating the hemispheric 427 lateralisation of low-level implicit measures of agency.

\section{$428 \quad 4.5$. Limitations}

429 The results of experiment 3 by themselves could not distinguish between an effect of anodal 430 stimulation of the putative left AG from an effect of cathodal stimulation of the putative right AG. 431 However, this result does allow us to exclude a model in which tDCS simply acts to increase neural noise, irrespective of polarity. Moreover, our experiment 1 found some evidence of a left-hemisphere 
anodal tDCS effect, while our experiment 2 found no evidence of any cathodal effect (though in the

left hemisphere, rather than the right). Cathodal stimulation effects in cognitive tasks are reported to be weak (Jacobson, Koslowsky, et al., 2012). Therefore, we provisionally favour an interpretation of experiment 3 based on a left parietal anodal effect, rather than a right-hemisphere cathodal effect. Further research would be required to draw a definitive conclusion.

Our study is further limited because we did not control for cases of crossmodal binding in the absence of active movement. Therefore, we cannot exclude the possibility that AG stimulation influenced some general feature of time perception, as opposed to temporal processing specific to agency. However, several studies have shown stronger binding between voluntary actions and outcomes than between other, similarly paired, events, including involuntary movements and outcomes (Engbert, Wohlschläger, Thomas, \& Haggard, 2007; Haggard et al., 2002) or pairs of sensory stimuli (Haggard, Aschersleben, Gehrke, \& Prinz, 2002; Haggard, Martin, Taylor-Clarke, Jeannerod, \& Franck, 2003). Moreover, other studies have investigated effects of parietal tDCS on time perception in general, in the absence of action: and these studies found no effect (Woods et al., 2014). Thus, the weight of other studies suggests that the intentional binding phenomenon reflects a distortion of perceptual timing that is, at least partly, specific to voluntary action.

\subsection{Dissociation between action binding and tone binding}

Anodal stimulation over putative left AG was a common condition in all 3 experiments. Accordingly, we could perform a pooled analysis of intentional binding results to compare this to the sham stimulations that were also included in each experiment. This analysis showed a highly significant reduction in tone binding with anodal stimulation of the putative left AG. We found no overall effect on action binding. Dissociations between action binding and tone binding have been reported previously (Wolpe et al., 2013), so it is possible that left parietal cortex is concerned primarily with tone binding, rather than with action binding. This conclusion would be consistent with previous studies suggesting that the AG processes mismatches in action outcomes (Farrer et al., 2008). On the other hand, recent studies of explicit agency judgement suggest that AG also processes prospective, 
462 463

premotor information arising during action selection (Chambon, Moore, \& Haggard, 2014; Chambon 461 et al., 2013).

Both online prospective and retrospective processes contribute to the intentional binding phenomenon (Moore and Haggard, 2008). The experimental design used here cannot identify the independent contribution of each process. However, binding of action towards outcome may rely more on prospective processes during action selection, while perceptual shift of outcome toward action may depend on retrospective, more inferential processes, triggered by reafferent signals about action outcome (Chambon, Moore, \& Haggard, 2014). Future studies may address this issue by designing new paradigms which dissociate prospective and retrospective components of agency and examine the role of $\mathrm{dLPFC}$ and $\mathrm{AG}$ in each of these components.

\subsection{Conclusion and clinical implications}

Sense of agency is an important and distinctive feature of human voluntary action. We used a causal intervention (tDCS) and an implicit perceptual measure of sense of agency (intentional binding) to examine the role of different brain areas in sense of agency. Anodal stimulation of parietal cortex consistently reduced the binding of tones towards actions. We hypothesised that the angular gyrus might contribute to the sense of agency by monitoring the linkage of actions to outcomes, or, alternatively and equivalently, failures of such linkage. Anodal stimulation of this area may correspond to artificial boosting of a mismatch detection process.

Sense of agency is altered following several classes of psychiatric and neurological disorders. In particular, patients with apraxia following lesions to the left parietal fail to recognise the source of a viewed manual gesture (Sirigu, Daprati, Pradat-Diehl, Franck, \& Jeannerod, 1999). This deficit is formally equivalent to an overestimation of agency in an explicit judgement task, consistent with damage to a neural centre detecting mismatches. The posterior form of 'alien hand syndrome' is also associated with contralateral parietal lesions (e.g., (Kloesel, Czarnecki, Muir, \& Keller, 2010)). Interestingly, these patients show involuntary and spontaneous movements of the contralateral limb, but may correctly perceive that they are not agents over these actions. The capacity for voluntary 
movement is often preserved. Quantitative assessment of the implicit sense of agency in parietal

487 patients would be of considerable value in understanding the neural basis of sense of agency.

489 Competing interests

490 The authors declare no competing financial interests.

\section{$491 \quad$ Acknowledgements}

492 This work was supported by the European Research Council Advanced Grant HUMVOL. PH was

493 additionally supported by a Professorial Research Fellowship from the ESRC. The funding sources

494 had no involvement, in study design; in the collection, analysis or interpretation of data; in the writing

495 of the report; or in the decision to submit the article for publication.

496 We thank Andrea Desantis for helpful comments during the preparation of the manuscript. 
Bardi, L., Kanai, R., Mapelli, D., \& Walsh, V. (2013). Direct current stimulation (tDCS) reveals parietal asymmetry in local/global and salience-based selection. Cortex, 49(3), 850-860. http://doi.org/10.1016/j.cortex.2012.04.016

Brass, M., \& Haggard, P. (2007). To do or not to do: the neural signature of self-control. The Journal of Neuroscience: The Official Journal of the Society for Neuroscience, 27(34), 9141-9145. http://doi.org/10.1523/JNEUROSCI.0924-07.2007

Buxbaum, L. J., Sirigu, A., Schwartz, M. F., \& Klatzky, R. (2003). Cognitive representations of hand posture in ideomotor apraxia. Neuropsychologia, 41(8), 1091-1113.

Cardinal, R. N., \& Aitken, M. R. F. (2013). ANOVA for the Behavioral Sciences Researcher. Psychology Press.

Chambon, V., Moore, J. W., \& Haggard, P. (2014). TMS stimulation over the inferior parietal cortex disrupts prospective sense of agency. Brain Structure \& Function. http://doi.org/10.1007/s00429-014-0878-6

Chambon, V., Wenke, D., Fleming, S. M., Prinz, W., \& Haggard, P. (2013). An Online Neural Substrate for Sense of Agency. Cerebral Cortex, 23(5), 1031-1037. http://doi.org/10.1093/cercor/bhs059

Cohen Kadosh, R., Soskic, S., Iuculano, T., Kanai, R., \& Walsh, V. (2010). Modulating neuronal activity produces specific and long-lasting changes in numerical competence. Current Biology: CB, 20(22), 2016-2020. http://doi.org/10.1016/j.cub.2010.10.007

Cravo, A. M., Claessens, P. M. E., \& Baldo, M. V. C. (2009). Voluntary action and causality in temporal binding. Experimental Brain Research, 199(1), 95-99. http://doi.org/10.1007/s00221-009-1969-0

Cumming, G. (2005). Understanding the Average Probability of Replication Comment on Killeen (2005). Psychological Science, 16(12), 1002-1004. http://doi.org/10.1111/j.14679280.2005.01650.x 
De Jong, B. M. (2011). Neurology of widely embedded free will. Cortex; a Journal Devoted to the Study of the Nervous System and Behavior, 47(10), 1160-1165. http://doi.org/10.1016/j.cortex.2011.06.011

Desmurget, M., Reilly, K. T., Richard, N., Szathmari, A., Mottolese, C., \& Sirigu, A. (2009). Movement intention after parietal cortex stimulation in humans. Science (New York, N.Y.), 324(5928), 811-813. http://doi.org/10.1126/science.1169896

Engbert, K., Wohlschläger, A., Thomas, R., \& Haggard, P. (2007). Agency, subjective time, and other minds. Journal of Experimental Psychology. Human Perception and Performance, 33(6), 1261-1268. http://doi.org/10.1037/0096-1523.33.6.1261

Farrer, C., Franck, N., Georgieff, N., Frith, C. D., Decety, J., \& Jeannerod, M. (2003). Modulating the experience of agency: a positron emission tomography study. NeuroImage, 18(2), 324-333.

Farrer, C., Frey, S. H., Horn, J. D. V., Tunik, E., Turk, D., Inati, S., \& Grafton, S. T. (2008). The Angular Gyrus Computes Action Awareness Representations. Cerebral Cortex, 18(2), 254261. http://doi.org/10.1093/cercor/bhm050

Farrer, C., \& Frith, C. D. (2002). Experiencing oneself vs another person as being the cause of an action: the neural correlates of the experience of agency. NeuroImage, 15(3), 596-603. http://doi.org/10.1006/nimg.2001.1009

Fink, G. R., Marshall, J. C., Halligan, P. W., Frith, C. D., Driver, J., Frackowiak, R. S., \& Dolan, R. J. (1999). The neural consequences of conflict between intention and the senses. Brain: A Journal of Neurology, 122 ( Pt 3), 497-512.

Fletcher, P. C., \& Frith, C. D. (2009). Perceiving is believing: a Bayesian approach to explaining the positive symptoms of schizophrenia. Nature Reviews. Neuroscience, 10(1), 48-58. http://doi.org/10.1038/nrn2536

Frith, C. D. (2014). Action, agency and responsibility. Neuropsychologia, 55, 137-142. http://doi.org/10.1016/j.neuropsychologia.2013.09.007

Frith, C. D., Blakemore, \& Wolpert, D. M. (2000). Abnormalities in the awareness and control of action. Philosophical Transactions of the Royal Society of London. Series B: Biological Sciences, 355(1404), 1771-1788. http://doi.org/10.1098/rstb.2000.0734 
Haggard, P., Aschersleben, G., Gehrke, J., \& Prinz, W. (2002). Action, binding, and awareness. In W. Prinz \& B. Hommel (Eds.), Common Mechanisms in Perception and Action B. (Vol. 19, pp. 266-285). OXFORD UNIVERSITY PRESS. Retrieved from http://eprints.ucl.ac.uk/4192/

Haggard, P., Clark, S., \& Kalogeras, J. (2002). Voluntary action and conscious awareness. Nature Neuroscience, 5(4), 382-385. http://doi.org/10.1038/nn827

Haggard, P., Martin, F., Taylor-Clarke, M., Jeannerod, M., \& Franck, N. (2003). Awareness of action in schizophrenia. Neuroreport, 14(7), 1081-1085. http://doi.org/10.1097/01.wnr.0000073684.00308.c0

Hecht, D., Walsh, V., \& Lavidor, M. (2010). Transcranial Direct Current Stimulation Facilitates Decision Making in a Probabilistic Guessing Task. The Journal of Neuroscience, 30(12), 4241-4245. http://doi.org/10.1523/JNEUROSCI.2924-09.2010

Horvath, J. C., Forte, J. D., \& Carter, O. (2014). Evidence that transcranial direct current stimulation (tDCS) generates little-to-no reliable neurophysiologic effect beyond MEP amplitude modulation in healthy human subjects: A systematic review. Neuropsychologia, 66C, 213236. http://doi.org/10.1016/j.neuropsychologia.2014.11.021

Jacobson, L., Goren, N., Lavidor, M., \& Levy, D. A. (2012). Oppositional transcranial direct current stimulation (tDCS) of parietal substrates of attention during encoding modulates episodic memory. Brain Research, 1439, 66-72. http://doi.org/10.1016/j.brainres.2011.12.036

Jacobson, L., Koslowsky, M., \& Lavidor, M. (2012). tDCS polarity effects in motor and cognitive domains: a meta-analytical review. Experimental Brain Research, 216(1), 1-10. http://doi.org/10.1007/s00221-011-2891-9

Jahanshahi, M., Jenkins, I. H., Brown, R. G., Marsden, C. D., Passingham, R. E., \& Brooks, D. J. (1995). Self-initiated versus externally triggered movements. I. An investigation using measurement of regional cerebral blood flow with PET and movement-related potentials in normal and Parkinson's disease subjects. Brain: A Journal of Neurology, 118 ( Pt 4), 913933. 
Kloesel, B., Czarnecki, K., Muir, J. J., \& Keller, A. S. (2010). Sequelae of a left-sided parietal stroke: posterior alien hand syndrome. Neurocase, 16(6), 488-493. http://doi.org/10.1080/13554794.2010.497154

Kranick, S. M., \& Hallett, M. (2013). Neurology of volition. Experimental Brain Research, 229(3), 313-327. http://doi.org/10.1007/s00221-013-3399-2

Krzanowski, W. J. (2000). Principles of Multivariate Analysis: A User's Perspective (2 edition). Oxford Oxfordshire ; New York: OUP Oxford.

Kühn, S., Brass, M., \& Haggard, P. (2013). Feeling in control: Neural correlates of experience of agency. Cortex, 49(7), 1935-1942. http://doi.org/10.1016/j.cortex.2012.09.002

Lee, W., \& Reeve, J. (2013). Self-determined, but not non-self-determined, motivation predicts activations in the anterior insular cortex: an fMRI study of personal agency. Social Cognitive and Affective Neuroscience, 8(5), 538-545. http://doi.org/10.1093/scan/nss029

Meier, U. (2006). A note on the power of Fisher's least significant difference procedure. Pharmaceutical Statistics, 5(4), 253-263. http://doi.org/10.1002/pst.210

Moore, J. W., Middleton, D., Haggard, P., \& Fletcher, P. C. (2012). Exploring implicit and explicit aspects of sense of agency. Consciousness and Cognition, 21(4), 1748-1753. http://doi.org/10.1016/j.concog.2012.10.005

Moore, J. W., \& Obhi, S. S. (2012). Intentional binding and the sense of agency: a review. Consciousness and Cognition, 21(1), 546-561. http://doi.org/10.1016/j.concog.2011.12.002

Moore, J. W., Ruge, D., Wenke, D., Rothwell, J., \& Haggard, P. (2010). Disrupting the experience of control in the human brain: pre-supplementary motor area contributes to the sense of agency. Proceedings. Biological Sciences / The Royal Society, 277(1693), 2503-2509. http://doi.org/10.1098/rspb.2010.0404

Moretto, G., Walsh, E., \& Haggard, P. (2011). Experience of agency and sense of responsibility. Consciousness and Cognition, 20(4), 1847-1854. http://doi.org/10.1016/j.concog.2011.08.014

Nathan, S. S., Sinha, S. R., Gordon, B., Lesser, R. P., \& Thakor, N. V. (1993). Determination of current density distributions generated by electrical stimulation of the human cerebral cortex. 
Electroencephalography and Clinical Neurophysiology, 86(3), 183-192. http://doi.org/10.1016/0013-4694(93)90006-H

Nitsche, M. A., Cohen, L. G., Wassermann, E. M., Priori, A., Lang, N., Antal, A., Pascual-Leone, A. (2008). Transcranial direct current stimulation: State of the art 2008. Brain Stimulation, 1(3), 206-223. http://doi.org/10.1016/j.brs.2008.06.004

Nitsche, M. A., Liebetanz, D., Lang, N., Antal, A., Tergau, F., \& Paulus, W. (2003). Safety criteria for transcranial direct current stimulation (tDCS) in humans. Clinical Neurophysiology: Official Journal of the International Federation of Clinical Neurophysiology, 114(11), 22202222; author reply 2222-2223.

Nitsche, M. A., \& Paulus, W. (2000). Excitability changes induced in the human motor cortex by weak transcranial direct current stimulation. The Journal of Physiology, 527 Pt 3, 633-639.

Okamoto, M., Dan, H., Sakamoto, K., Takeo, K., Shimizu, K., Kohno, S., Dan, I. (2004). Threedimensional probabilistic anatomical cranio-cerebral correlation via the international 10-20 system oriented for transcranial functional brain mapping. NeuroImage, 21(1), 99-111. http://doi.org/10.1016/j.neuroimage.2003.08.026

Poreisz, C., Boros, K., Antal, A., \& Paulus, W. (2007). Safety aspects of transcranial direct current stimulation concerning healthy subjects and patients. Brain Research Bulletin, 72(4-6), 208214. http://doi.org/10.1016/j.brainresbull.2007.01.004

Rowe, J. B., Hughes, L., \& Nimmo-Smith, I. (2010). Action selection: a race model for selected and non-selected actions distinguishes the contribution of premotor and prefrontal areas. NeuroImage, 51(2), 888-896. http://doi.org/10.1016/j.neuroimage.2010.02.045

Sirigu, A., Daprati, E., Ciancia, S., Giraux, P., Nighoghossian, N., Posada, A., \& Haggard, P. (2004). Altered awareness of voluntary action after damage to the parietal cortex. Nature Neuroscience, 7(1), 80-84. http://doi.org/10.1038/nn1160

Sirigu, A., Daprati, E., Pradat-Diehl, P., Franck, N., \& Jeannerod, M. (1999). Perception of selfgenerated movement following left parietal lesion. Brain, 122(10), 1867-1874. http://doi.org/10.1093/brain/122.10.1867 
Sperduti, M., Delaveau, P., Fossati, P., \& Nadel, J. (2011). Different brain structures related to selfand external-agency attribution: a brief review and meta-analysis. Brain Structure \& Function, 216(2), 151-157. http://doi.org/10.1007/s00429-010-0298-1

Spitoni, G. F., Pireddu, G., Cimmino, R. L., Galati, G., Priori, A., Lavidor, M., Pizzamiglio, L. (2013). Right but not left angular gyrus modulates the metric component of the mental body representation: a tDCS study. Experimental Brain Research. Experimentelle Hirnforschung. Expérimentation Cérébrale, 228(1), 63-72. http://doi.org/10.1007/s00221-013-3538-9

Synofzik, M., Vosgerau, G., \& Newen, A. (2008). Beyond the comparator model: a multifactorial two-step account of agency. Consciousness and Cognition, 17(1), 219-239. http://doi.org/10.1016/j.concog.2007.03.010

Wolpe, N., Haggard, P., Siebner, H. R., \& Rowe, J. B. (2013). Cue integration and the perception of action in intentional binding. Experimental Brain Research, 229(3), 467-474. http://doi.org/10.1007/s00221-013-3419-2

Wolpert, D. M., \& Ghahramani, Z. (2000). Computational principles of movement neuroscience. Nature Neuroscience, 3 Suppl, 1212-1217. http://doi.org/10.1038/81497

Woods, A. J., Hamilton, R. H., Kranjec, A., Minhaus, P., Bikson, M., Yu, J., \& Chatterjee, A. (2014). Space, time, and causality in the human brain. NeuroImage, 92, 285-297. http://doi.org/10.1016/j.neuroimage.2014.02.015

Zwissler, B., Sperber, C., Aigeldinger, S., Schindler, S., Kissler, J., \& Plewnia, C. (2014). Shaping Memory Accuracy by Left Prefrontal Transcranial Direct Current Stimulation. The Journal of Neuroscience, 34(11), 4022-4026. http://doi.org/10.1523/JNEUROSCI.5407-13.2014 


\section{Captions}

Fig. 1. Schematic of intentional binding. Action and tone shifts are measured by subtracting each

671 participant's mean judgement error in baseline conditions from judgement error in operant conditions.

672 These shifts serve as measures of intentional binding. Vertical bars and thin arrows represent mean 673 judgement errors in each condition. Thick arrows represent binding effects. See text for full 674 explanation.

675 Fig. 2. A. tDCS montage and study design (anode +, cathode -). See text for explanation. In 676 Experiment 1, to control for the cutaneous sensation of all three locations, all sponges were kept in 677 place across the three sessions. However, only two of them were actually functioning in each session. 678 B. Stimulation protocol. The order of conditions was randomised within each session.

679 Fig. 3. Intentional binding in Experiment 1. A) The dashed line indicates the perceived time of either 680 action or tone in the corresponding baseline condition. A separate baseline condition was used for 681 each session, and differences in baseline values across sessions have been removed for display 682 purposes. Binding effects are drawn to scale, and values are in milliseconds. B) Mean binding effects 683 in ms. The sign of tone binding effects has been inverted to allow for comparison with action binding. 684 Error bars show standard error of the mean. ${ }^{*} \mathrm{p}<0.05$.

685 Fig. 4. Intentional binding in Experiment 2. Format as in Fig. 3.

686 Fig. 5. Intentional binding in Experiment 3. Format as in Fig. 3. 
- Effects of tDCS on implicit measures of sense of agency were measured.

- Anodal stimulation of the left angular gyrus decreased 'intentional binding'.

- Cathodal stimulation had no effect.

- The left angular gyrus plays a key role in the experience of agency. 
Click here to download high resolution image

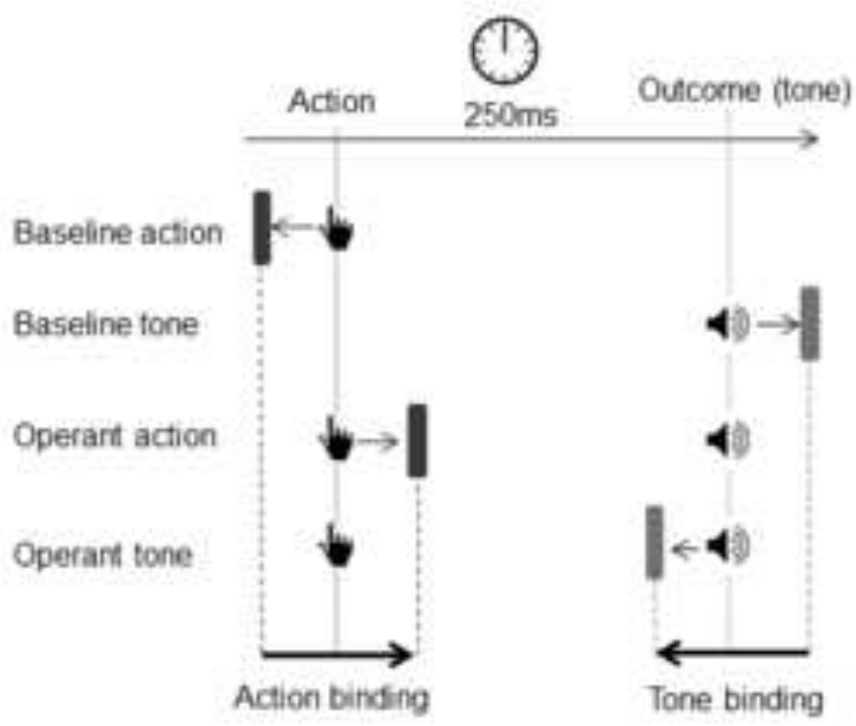


Click here to download high resolution image

A.

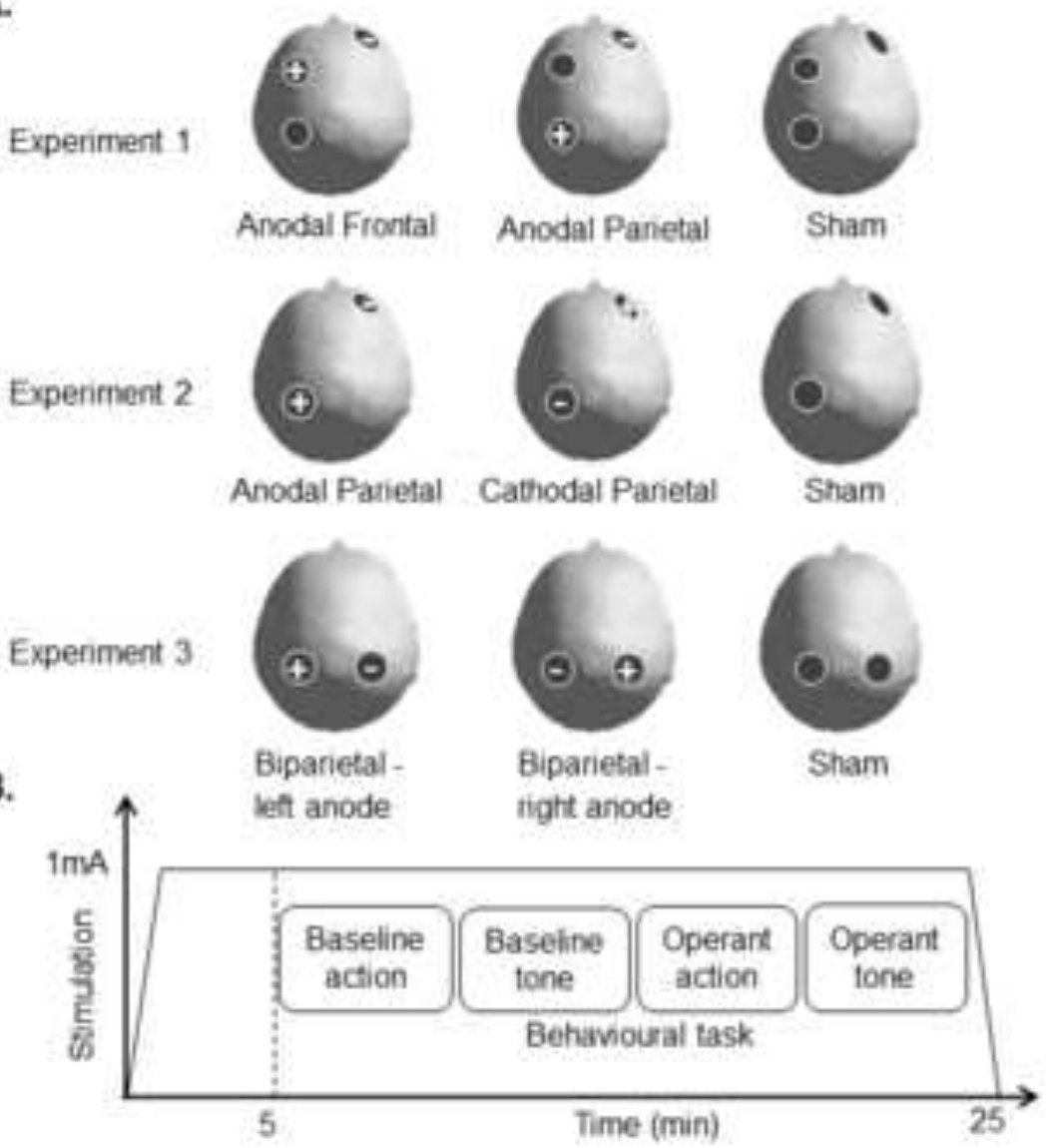


Click here to download high resolution image

A.

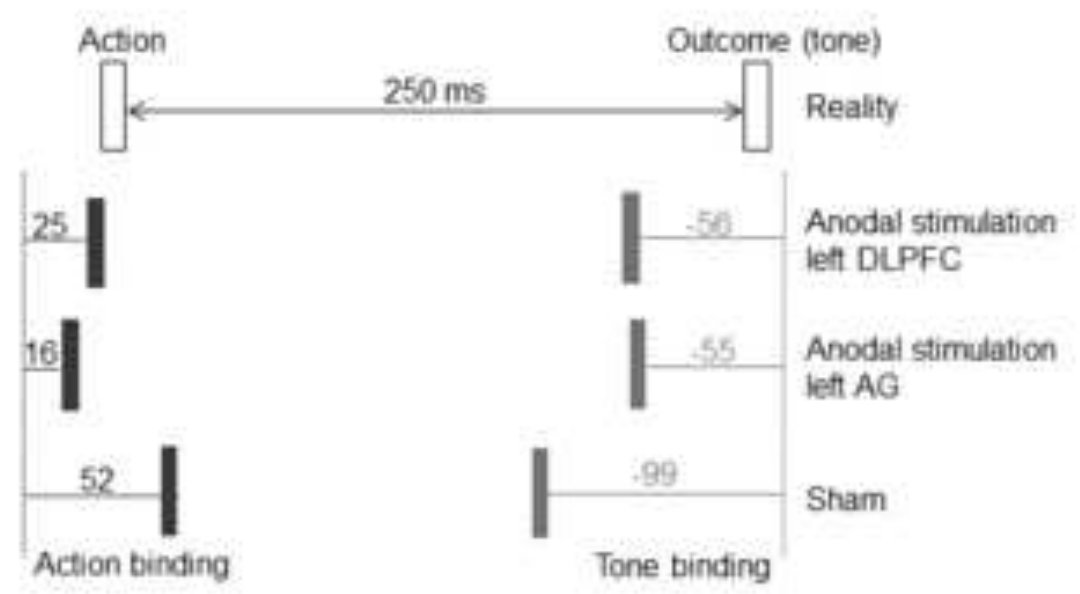

B.

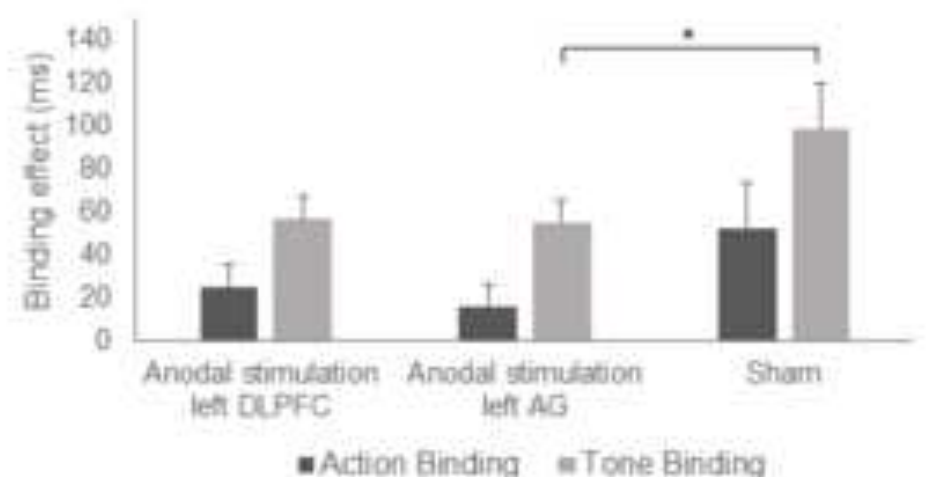

-Action Binding =Tone Binding 
Click here to download high resolution image

A.

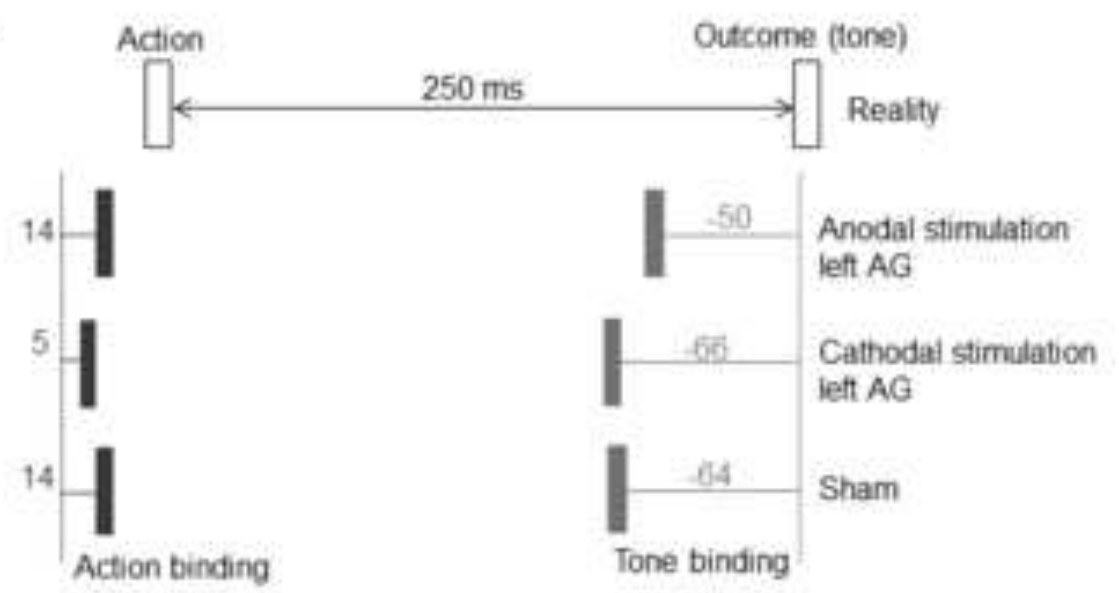

B.

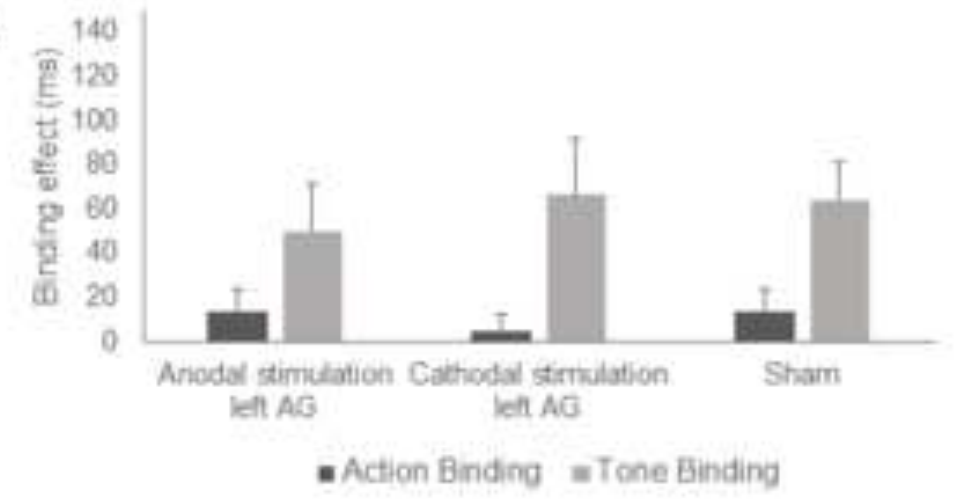


Click here to download high resolution image

A.

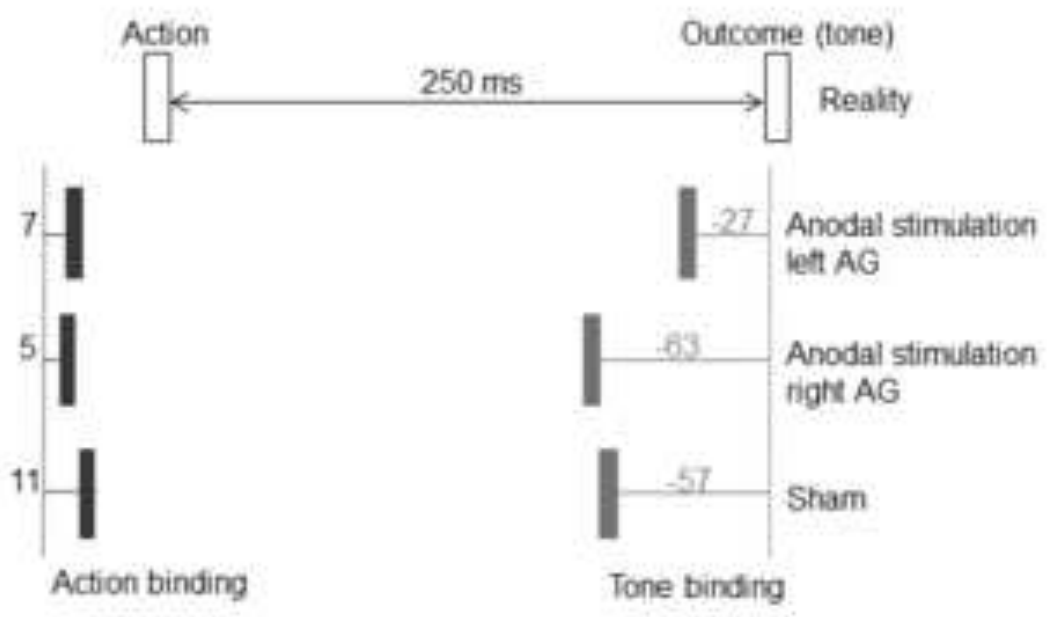

B.

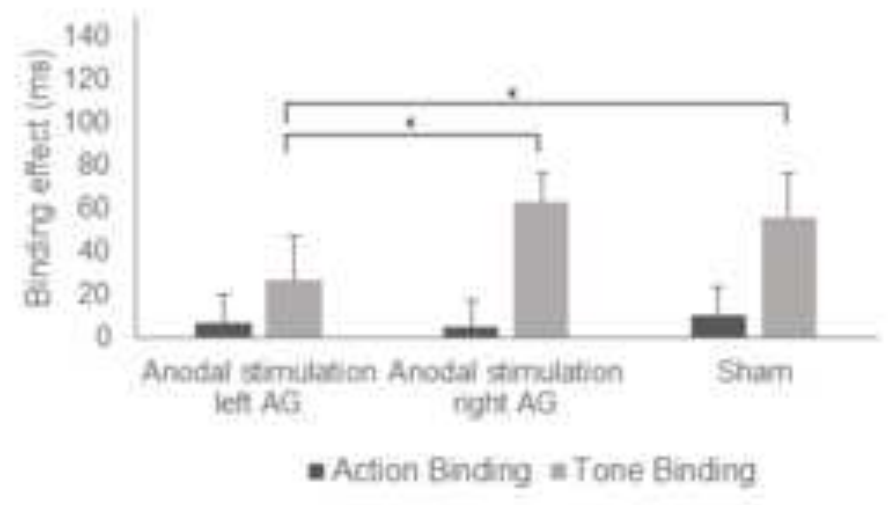


Suppl. material A
Click here to download Suppl. material for online publication only: Supplementary_CORTEX_A.pdf Click here to download Suppl. material for online publication only: Supplementary_CORTEX_A.pdf

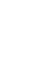

(1)

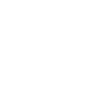
.

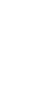
Click here to download Suppl. material for online publication only: Supplementary_CORTEX_A.pdf (1) (1) (1) 
Suppl. material B
Click here to download Suppl. material for online publication only: Supplementary_CORTEX_B.pdf (a)

(1)

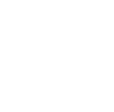

更

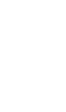
(20) 